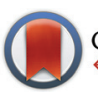

CrossMark

\& click for updates

Cite this: Polym. Chem., 2016, 7, 184

\title{
Processing and adjusting the hydrophilicity of poly(oxymethylene) (co)polymers: nanoparticle preparation and film formation $\uparrow$
}

\author{
Markus B. Bannwarth, $t^{\mathrm{a}, \mathrm{c}}$ Rebecca Klein, $\xi^{\mathrm{b}, \mathrm{c}}$ Sven Kurch, ${ }^{\mathrm{b}}$ Holger Frey, ${ }^{\mathrm{b}}$ \\ Katharina Landfester ${ }^{a}$ and Frederik R. Wurm ${ }^{* a}$
}

\begin{abstract}
Handling the insoluble POM: the preparation of nanoparticles based on hyperbranched-linear-hyperbranched ABA triblock copolymers with variable hydrophilicity and composed of short hyperbranched polyglycerol (hbPG) as the A-blocks and linear poly(oxymethylene) (POM) as a B-block is described. The POM-hbPG-nanoparticles with diameters in the range of 190 to $250 \mathrm{~nm}$ were generated in a convenient process, combining the solvent evaporation process with the miniemulsion technique, a water borne handling for POM-copolymers. Furthermore, the film formation properties of the nanoparticles were investigated by deposition on silicon and subsequent sintering, which leads to films with a thickness in the $\mu \mathrm{m}$-range that were investigated via SEM. The surface properties of these films were investigated via static contact angle measurements at the liquid/vapor interface. The contact angle decreases from $67^{\circ}$ for the polymer film based on POM with two hydroxyl end groups to $29^{\circ}$ for POM-copolymers with 16 hydroxyl groups, confirming the influence of the polymer structure and size of the hbPG block on the surface properties. In summary, this work presents a possibility for a facile handling and film formation of the insoluble POM, opening new applications, e.g., in coatings.
\end{abstract}

Received 3rd September 2015 Accepted 20th October 2015 DOI: $10.1039 / c 5 p y 01418 b$ www.rsc.org/polymers greatly improved thermal stability is achieved at the expense of a slightly reduced degree of crystallinity. ${ }^{1-4}$

To the best of our knowledge, organic or aqueous dispersions of POM nanoparticles have not been prepared to date, although this may be interesting for handling and processing of POM. Several groups have prepared nanocomposites based on POM and inorganic nanoparticles to ameliorate the properties of POM. Romero-Ibarra et $a .^{5}$ blended POM with $\mathrm{BaSO}_{4}$ nanoparticles to obtain X-ray opaque materials. The combination with polyhedral oligomeric silsesquioxanes (POSS) resulted in enhanced thermal stability. ${ }^{6,7}$ Due to the flame-retardant and antioxidant properties and its brightness, nanocomposites with $\mathrm{TiO}_{2}$ nanoparticles led to higher thermal stability. $^{8}$ Combination of POM with hydroxyapatite nanoparticles permits the use for bone tissue replacement. ${ }^{9-11}$ Furthermore, hybrid systems based on $\mathrm{MoS}_{2},{ }^{12-14} \mathrm{Al}_{2} \mathrm{O}_{3}$, ${ }^{15,16}$ $\mathrm{ZnO},{ }^{17,18}$ and clay ${ }^{19,20}$ nanoparticles and POM were produced.

In this work, polymer nanoparticles based on POM (co)polymers have been prepared. A versatile method for the generation of a variety of nanoparticles is the miniemulsion technique. This technique enables the formation of structured polymeric nanoparticles and the encapsulation of solid or liquid, organic or inorganic or hydrophilic or hydrophobic materials into a polymeric carrier. ${ }^{21}$ In a typical oil-in-water miniemulsion, an oil, a hydrophobic agent, an emulsifier and 
water are homogenized by high shear forces, resulting in homogeneous and monodisperse droplets in the size range of 30 to $500 \mathrm{~nm}^{22}$ For particle formation with preformed polymers a combination of the emulsion/solvent evaporation method $^{23}$ and the miniemulsion technique can be used. In this case, the droplets consist of a solution of the preformed polymer and after evaporation of the solvent, a polymer dispersion is obtained. ${ }^{24-26}$

Recently reported ${ }^{28}$ preformed nonlinear ABA triblock copolymers consisting of a linear POM block and hyperbranched poly(glycerol) (hbPG) blocks are used for the miniemulsion/ solvent evaporation protocol to generate nanoparticles consisting of hbPG- $b$-POM- $b$-hbPG copolymers. Various degrees of polymerization of hbPG were studied with respect to the hydrophilicity of the resulting polymeric nanoparticles. The particle dispersion was drop-cast and sintered to smooth films onto a silicon surface and investigated via static contact angle measurements. A strong impact of the hbPG-segments on the hydrophilicity of the POM surface was detected. The approach allows facile handling and processing of the highly crystalline and therefore insoluble POM as an alternative to injection molding and extrusion. We demonstrate that sintering results in films that retain the excellent mechanical properties of POM, which is of significant interest for impact resistant surfaces.

\section{Experimental}

\section{Instrumentation}

${ }^{1} \mathrm{H}$ NMR spectra were recorded at $600 \mathrm{MHz}$ at $37{ }^{\circ} \mathrm{C}$ on a Bruker Avance III and are referenced internally to residual proton signals of the deuterated solvent. SEC measurements in HFIP containing $0.05 \mathrm{~mol} \mathrm{~L}^{-1}$ KFAc were performed on a Jasco LC-NetII/ADC as an integrated instrument including a PS PFG 100 A column and a RI detector. Poly(methyl methacrylate) provided by Polymer Standards Service was used as calibration standard. DSC measurements were carried out on a PerkinElmer DSC 8500 in the temperature range of -95 to $180{ }^{\circ} \mathrm{C}$ in two heating runs, using heating rates of $10 \mathrm{~K} \mathrm{~min}^{-1}$ under nitrogen. The hydrodynamic radius of the POM-nanoparticles was determined via DLS measurements on a NICOMP Zetasizer at a measurement angle of $90^{\circ}$. The dispersion obtained after particle formation was diluted with cyclohexane $(1: 50)$ and measured at $25{ }^{\circ} \mathrm{C}$. Scanning electron microscopy (SEM) was performed on a Hitachi SU8000 at an extractor voltage of $3.0 \mathrm{kV}$. To form a miniemulsion, a 1/2 inch tip Branson Sonifier W-450-Digital was used. Contact angle measurements were performed on a Dataphysics Contact Angle System OCA using MilliQ-water as interface agent.

\section{Materials}

Materials were used as received, if not stated otherwise. Trioxane, 1,3-dioxolane and triflic acid were obtained from Ticona $\mathrm{GmbH}$. Cesium hydroxide monohydrate and 1,1,1,3,3,3-hexafluoro-2-isopropanol- $d_{2}$ (HFIP- $d_{2}$ ) were purchased from Acros. Methanol, cyclohexane, benzene and sodium dodecyl sulfate (SDS) were obtained from Sigma-Aldrich and HFIP from Apollo Scientific Limited. Glycidol and dimethylacetamide (DMAc) (99\% Acros) were purified by distillation from $\mathrm{CaH}_{2}$ prior to use. The surfactant poly[(ethylene-co-butylene)- $b$-(ethylene oxide)] with $M_{\mathrm{w}}=3700 \mathrm{~g} \mathrm{~mol}{ }^{-1}$ for $\mathrm{P}(\mathrm{E} / \mathrm{B})$ and $M_{\mathrm{w}}=3600 \mathrm{~g}$ $\mathrm{mol}^{-1}$ for PEO was synthesized as described elsewhere. ${ }^{27}$

\section{Synthesis of poly(oxymethylene) (POM) and the ABA triblock copolymers (hbPG- $b$-POM- $b$-hbPG)}

The synthesis of POM and the corresponding ABA triblock copolymers was performed according to literature. ${ }^{28}$ Characterization data of the linear poly(oxymethylene) (co)polymer block: SEC (HFIP, PMMA-Std.): $M_{\mathrm{n}}=10700 \mathrm{~g} \mathrm{~mol}^{-1}$; PDI = 2.09. ${ }^{1} \mathrm{H}$ NMR (HFIP- $\left.d_{2}, 600 \mathrm{MHz}\right): \delta[\mathrm{ppm}]=5.20-5.00\left(-\mathrm{CH}_{2}\right.$ polymer main chain); 5.00-4.95 $\left(-\mathrm{CH}_{2}-\right.$ dioxolane); 3.95-3.90 $\left(-\mathrm{CH}_{2}-\right.$ dioxolane), yield: $53 \%$.

For the synthesis of the triblock copolymers, the linear bishydroxy-functional POM macroinitiator was deprotonated and a solution of glycidol was slowly added with a syringe pump to perform the hypergrafting reactions on both ends. The characterization data of the obtained polymers are summarized in Table 1.

\section{Preparation of poly(oxymethylene) (POM) and POM-copolymer nanoparticles}

For the preparation of the nanoparticles, $50 \mathrm{mg}$ of the respective POM (co)-polymers were dissolved in $2 \mathrm{~g}$ of HFIP at $30{ }^{\circ} \mathrm{C}$ in an ultrasonication bath. Separately, $10 \mathrm{mg}$ of the surfactant poly[(ethylene-co-butylene)- $b$-(ethylene oxide)] was dissolved in $10 \mathrm{~g}$ cyclohexane at $40^{\circ} \mathrm{C}$ in an ultrasonication bath.

Table 1 Characterization data for nonlinear copolymers

\begin{tabular}{|c|c|c|c|c|c|c|c|c|}
\hline No. & Composition (NMR) & $\mathrm{POM} / \mathrm{mol} \%$ & $\mathrm{PG} / \mathrm{mol} \%$ & $M_{\mathrm{n}}{ }^{a} / \mathrm{g} \mathrm{mol}^{-1}$ & $M_{\mathrm{n}}^{b} / \mathrm{g} \mathrm{mol}^{-1}$ & $M_{\mathrm{w}} / M_{\mathrm{n}}^{b}$ & $T_{\mathrm{m}}{ }^{c} /{ }^{\circ} \mathrm{C}$ & $T_{\mathrm{g}}{ }^{c} /{ }^{\circ} \mathrm{C}$ \\
\hline 1 & $\mathrm{POM}_{120}{ }^{d}$ & 100 & 0 & 3800 & 10700 & 2.09 & 164.4 & - \\
\hline 2 & $\mathrm{hbPG}_{2}-b-\mathrm{POM}_{120}-b-\mathrm{hbPG}_{2}$ & 97 & 3 & 4000 & 11700 & 1.96 & 159.3 & -65.3 \\
\hline 3 & $\mathrm{hbPG}_{3}-b-\mathrm{POM}_{120}-b-\mathrm{hbPG}_{3}$ & 95 & 5 & 4200 & 14600 & 1.82 & 159.3 & - \\
\hline 4 & $\mathrm{hbPG}_{5}-b-\mathrm{POM}_{120}-b-\mathrm{hbPG}_{5}$ & 92 & 8 & 4400 & 14400 & 1.88 & 157.6 & -62.1 \\
\hline 5 & $\mathrm{hbPG}_{7}-b-\mathrm{POM}_{120}-b-\mathrm{hbPG}_{7}$ & 89 & 11 & 4800 & 10000 & 2.53 & 159.0 & -55.0 \\
\hline
\end{tabular}

${ }^{a}$ Calculated from ${ }^{1} \mathrm{H}$ NMR spectra. ${ }^{b}$ Determined by SEC in HFIP (RI-detector signal, PMMA standards). ${ }^{c}$ DSC data from second heating run, heating rate: $10 \mathrm{~K} \mathrm{~min}^{-1} .{ }^{d}$ Copolymer of trioxane and dioxolane $(10 \%)$. 
Both phases were mixed, pre-emulsified mechanically and sonified for 2 min under ice cooling using a 1/2 inch tip sonifier ( 5 s pulse, $10 \mathrm{~s}$ pause, $70 \%$ amplitude). The resulting miniemulsion was stirred for $30 \mathrm{~min}$ at $600 \mathrm{rpm}$ in an open vial to evaporate the HFIP. Purification of excess surfactant was achieved by centrifugation of the nanoparticles and redispersion in pure cyclohexane. For redispersion in water, $0.5 \mathrm{~g}$ of the nanoparticle dispersion in cyclohexane was added to $10 \mathrm{~g}$ of an aqueous solution containing $10 \mathrm{mg}$ of SDS and the two phase system stirred in an open vial for $4 \mathrm{~h}$ at $1400 \mathrm{rpm}$ to evaporate the cyclohexane.

\section{Film formation}

For film formation, the nanoparticle dispersion in cyclohexane (solid content of $1 \mathrm{wt} \%$ ) was drop-casted onto a silicon wafer. Heating of the wafer for $10 \mathrm{~s}$ to $180{ }^{\circ} \mathrm{C}$ resulted in film formation of the POM-particles. To analyze the film consistency and thickness, the wafer was broken in half and investigated via SEM under various angles.

\section{Results and discussion}

\section{Polymer synthesis and characterization}

In this work, the established linear POM homopolymer as well as innovative hyperbranched-linear-hyperbranched ABA triblock copolymers based on hbPG and POM were applied for the preparation of aqueous nanoparticle dispersions. As summarized in Table 1, the polymers mainly consist of POM (89 to $97 \mathrm{~mol} \%$ ) and only a small amount of hbPG (3 to $11 \mathrm{~mol} \%$ ) was attached. By this, the mechanical properties of POM are rarely affected, however, the hypergrafting enabled an adjustable hydrophilicity of the polymers, nanoparticles, and the obtained polymer films.

The ABA triblock copolymers were synthesized via a combination of cationic ring-opening copolymerization (ROP), followed by the multibranching anionic ROP of glycidol. ${ }^{28}$ ABAtype nonlinear block copolymers have received increased attention in recent years, due to their high end group functionality based on the combination of a linear with a hyperbranched segment. ${ }^{29}$ In the first step, linear bishydroxy-functional poly(oxymethylene) (POM) copolymers were prepared by cationic ring-opening copolymerization of trioxane and 1,3-dioxolane with formic acid as a transfer agent. The resulting formiate end groups were hydrolyzed to obtain the bishydroxy end-functional POM. This serves as a difunctional macroinitiator for the ensuing hypergrafting reaction of glycidol, resulting in nonlinear ABA triblock copolymers with an adjustable number of hydroxyl groups (Fig. 1). ${ }^{30,31}$ To enable a clear differentiation between the linear POM and the hyperbranched block copolymers, the linear POM (consisting of trioxane and dioxolane) will be called POM homopolymer in the following.

Table 1 shows the characterization data of the polymers used for nanoparticle formation obtained by NMR spectroscopy and SEC as well as their thermal properties determined by DSC.

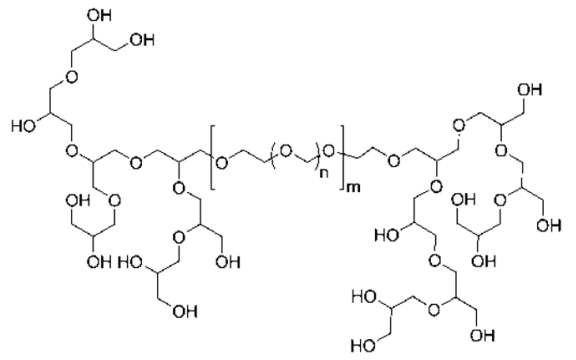

Fig. 1 Structure of hyperbranched-linear-hyperbranched ABA triblock copolymers. ${ }^{28}$

The number-averaged molecular weight of the difunctional macroinitiator (Fig. 1) was determined via ${ }^{1} \mathrm{H}$ NMR endgroup analysis (compare Fig. S1 $\dagger$ ). Integration of the resonances of the methylene signals stemming from ring-opened trioxane (at $5.10 \mathrm{ppm}$ ) and dioxolane (at 5.00 and $3.95 \mathrm{ppm}$ ) results in a $M_{\mathrm{n}}$ of $3800 \mathrm{~g} \mathrm{~mol}^{-1}$. SEC in HFIP vs. PMMA standards overestimates the molecular weights at $c a .10 \mathrm{~kg} \mathrm{~mol}^{-1}$, however, the molecular weight dispersity of $c a .2$ is comparable to previous reports. After hypergrafting of glycidol new signals between 3.50 and 4.20 ppm corresponding to hbPG indicate the successful triblock copolymer formation. ${ }^{28}$

The molecular weights (determined by NMR) of the resulting nonlinear triblock copolymers vary from 4000 to $4800 \mathrm{~g}$ $\mathrm{mol}^{-1}$. SEC measurements (Fig. S2 $\dagger$ ) determine apparent molecular weights in the range of 10000 to $14600 \mathrm{~g} \mathrm{~mol}^{-1}$ and moderate polydispersities $\left(M_{\mathrm{w}} / M_{\mathrm{n}}: 1.82-2.53\right)$.

Thermal properties were investigated via differential scanning calorimetry (DSC, compare the ESI $\dagger$ ). The characteristic melting range of $\mathrm{POM}$ is detected between $175^{\circ} \mathrm{C}$ and $185^{\circ} \mathrm{C}$ (only trioxane as a monomer) and around $165{ }^{\circ} \mathrm{C}$ for copolymers based on trioxane and dioxolane, strongly dependent on the dioxolane content, while reported glass transition temperatures $\left(T_{\mathrm{g}}\right)$ are detected at $-82{ }^{\circ} \mathrm{C} .{ }^{32}$ From the data in Table $1 \mathrm{a}$ melting temperature $\left(T_{\mathrm{m}}\right)$ of $164.4{ }^{\circ} \mathrm{C}$ was detected for the macroinitiator (1), which is in the expected range. For the triblock copolymers the $T_{\mathrm{m}} \mathrm{s}$ decrease to values of 157.6 to $159.3^{\circ} \mathrm{C}$. Additionally, a $T_{\mathrm{g}}$ is observable that increases from -65.3 to $-55.0^{\circ} \mathrm{C}$ with increasing hbPG content. For all block copolymers the $\mathrm{Tg}$ of the hbPG segments (with a typical $T_{\mathrm{g}}$ of ca. $-20{ }^{\circ} \mathrm{C}$ ) is not detectable, probably due to the rather low $\mathrm{DP}_{\mathrm{n}}$ of the hbPG segments and was reported earlier for other linear-hyperbranched block copolymers. ${ }^{33}$

\section{Nanoparticle preparation}

Controlled solvent evaporation combined with miniemulsion is a facile process to prepare polymer nanoparticles from previously synthesized materials by dissolving them in a good solvent for the polymer and dispersing this solution in a nonsolvent. After solvent evaporation, a polymer-nanoparticle dispersion is obtained. For the POM (co)polymers it was necessary to optimize this protocol due to the low solubility of POM in most organic solvents. Fluorinated solvents, such as HFIP 

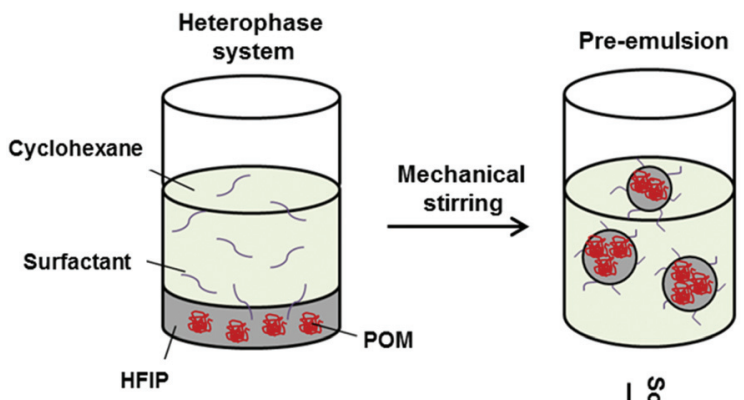

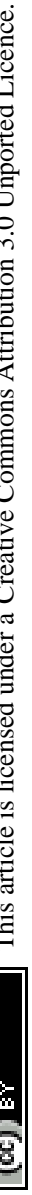

can be used to dissolve POM and its copolymers (Fig. 2). The POM (co)polymers were dissolved in HFIP and mechanical stirring was used to produce a pre-emulsion of HFIP/polymer droplets in a continuous cyclohexane phase. For stabilization of this emulsion a block copolymer consisting of a poly(ethylene oxide) block with $M_{\mathrm{w}} \sim 3600 \mathrm{~g} \mathrm{~mol}^{-1}$ and a poly(ethylene-cobutylene) block with $M_{\mathrm{w}} \sim 3700 \mathrm{~g} \mathrm{~mol}^{-1}$ was identified as an ideal nonionic surfactant. It is known to be suitable for waterin-cyclohexane (mini)emulsions and we identified it to be suitable also for HFIP-in-cyclohexane emulsions as well. The hydrophilic PEO will be located in the polar HFIP droplet phase, while the $\mathrm{P}(\mathrm{E} / \mathrm{B})$ block will be mainly present in the apolar phase to sterically stabilize the emulsion and prevent the droplets from coalescence. Sonication of the two-phase system leads to the formation of miniemulsion droplets of HFIP containing the POM homo- and block copolymers. By stirring the miniemulsion in an open vial at room temperature, the good solvent HFIP was evaporated quickly due to the low boiling point of HFIP of $c a .58{ }^{\circ} \mathrm{C}$. After evaporation of HFIP, a nanoparticles dispersion of POM (co)polymers in cyclohexane was obtained, which was stable over a period of several months.

The diameter of the POM and hbPG- $b$-POM- $b$-hbPG nanoparticles was found to be in the range of $190-250 \mathrm{~nm}$ with a standard deviation of $\sim 30 \%$ by dynamic light scattering (DLS) (Table 2). In all cases the nanoparticle diameters were similar and no effect of the copolymer composition, i.e. differences between POM and the nonlinear POM block copolymers were
Table 2 Hydrodynamic diameters of different POM nanoparticles determined via DLS

\begin{tabular}{llll}
\hline No. & Composition (NMR) & $\begin{array}{l}\text { Hydrodynamic } \\
\text { diameter/nm }\end{array}$ & $\begin{array}{l}\text { Standard } \\
\text { deviation }\end{array}$ \\
\hline 1 & $\mathrm{POM}_{120}$ & 220 & $28 \%$ \\
2 & $\mathrm{hbPG}_{2}-b-\mathrm{POM}_{120}-b-\mathrm{hbPG}_{2}$ & 250 & $27 \%$ \\
3 & $\mathrm{hbPG}_{3}-b-\mathrm{POM}_{120}-b-\mathrm{hbPG}_{3}$ & 190 & $38 \%$ \\
4 & $\mathrm{hbPG}_{5}-b-\mathrm{POM}_{120}-b-\mathrm{hbPG}$ & 210 & $26 \%$ \\
5 & $\mathrm{hbPG}_{7}-b-\mathrm{POM}_{120}-b-\mathrm{hbPG}_{7}$ & 200 & $21 \%$
\end{tabular}

observed. Thus, the size of the nanoparticles is independent of the number of hbPG-units at the ends, at least to an extent of in average seven PG-units at each chain end.

Additionally, redispersion of the organic nanoparticle dispersion (from cyclohexane) in water was possible using an aqueous sodium dodecylsulfate (SDS) solution (with subsequent dialysis), leading to an aqueous dispersion of POM nanoparticles with variable hydrophilicity. A slight increase of the nanoparticle sizes was found (300-320 nm, standard deviation $\sim 42 \%$, from DLS, $c f$. ESI Table S1 $\dagger$ ), probably due to swelling of the polymers in water.

To compare the sizes of the nanoparticles in solution and in the dried state and to get an insight into the morphology of deposited films from the POM homo- and block copolymers, SEM imaging of all samples was performed (Fig. 3 and S3†). The diameters determined from the SEM images are similar to the values determined by DLS, however, the average diameter is slightly smaller. As expected, spherical nanoparticles are obtained, however, a perfect spherical shape is not always found, and a slight anisotropy can be observed. A certain anisotropy of the nanoparticles is typical for nanoparticles consisting of polymers with a high degree of crystallinity, such as POM. ${ }^{34}$

Additionally, the polyacetal structure of the POM-block makes these nanoparticles also interesting as degradable materials for various applications. The acid catalyzed degradation of the nanoparticles was studied with an aqueous dis-

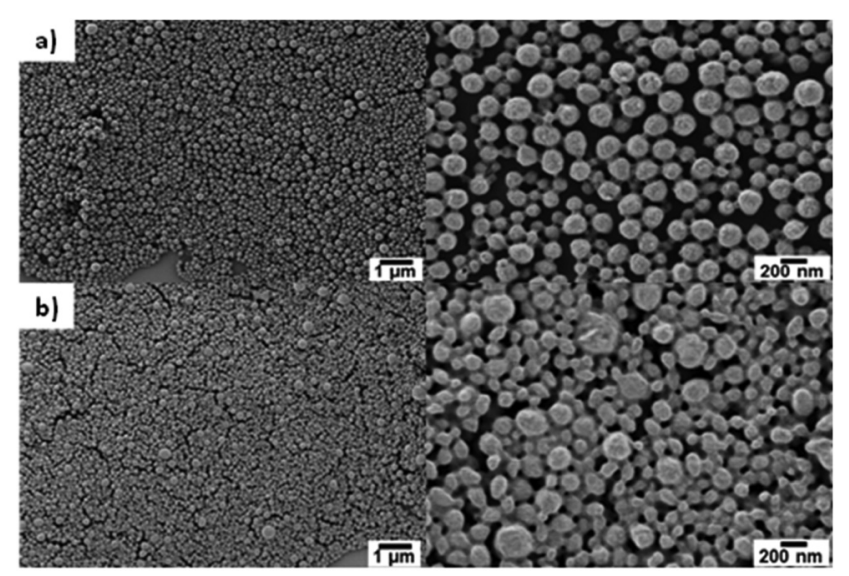

Fig. 3 SEM images of POM nanoparticles composed of POM and POM block copolymers with different end-group functionalization: (a) $\mathrm{POM}_{120}$, (b) $\mathrm{hbPG}_{5}-b-\mathrm{POM}_{120}-b-\mathrm{hbPG}_{5}$. 
persion. To this dispersion hydrochloric or acetic acid was added as a proof of principle and the mixture heated to $80^{\circ} \mathrm{C}$ for one hour. In both cases a clear solution was obtained revealing the full degradation of the nanoparticles under acidic conditions. Therefore, different materials like pigments or drugs can be encapsulated and can be released reducing the $\mathrm{pH}$. This opens plenty of new applications in, e.g., industry or medicine.

\section{Film formation}

For film formation, the POM nanoparticle dispersion was drop-casted on a silicon wafer and sintered at elevated temperatures (see Experimental section). In order to obtain impact resistant, homogeneous films, the particles have to be heated above their melting temperature $\left(T_{\mathrm{m}}\right)$, which is in the range of 158-165 ${ }^{\circ} \mathrm{C}$ (see Table 1) for POM and the POM copolymers. The surface of the films formed after annealing to $180^{\circ} \mathrm{C}$ for $10 \mathrm{~s}$ was investigated via SEM. Fig. 4 shows the particles after deposition on the silicon wafer before sintering (top view a, side view b) and after sintering (top view c, side view d). After sintering at $180{ }^{\circ} \mathrm{C}$ for 10 seconds a homogenous film is obtained, evidencing the suitability of these nanoparticles for the formation of smooth POM surfaces. The optical micrographs show the silicon wafer coated with $\mathrm{hbPG}_{3}-b-\mathrm{POM}_{120^{-}}-b$ $\mathrm{hbPG}_{3}$ nanoparticles before (e) and after (f) the sintering procedure. The deposited nanoparticles can be seen with the

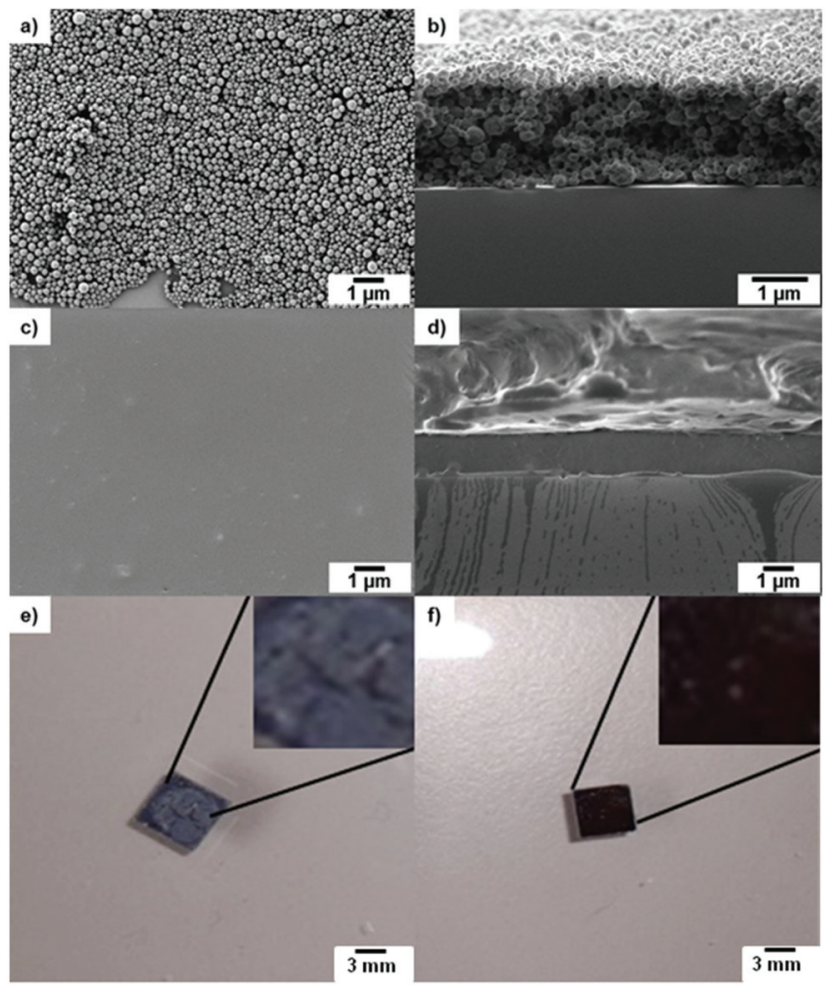

Fig. 4 SEM images of $h b \mathrm{PG}_{3}-b-\mathrm{POM}_{120}-b-\mathrm{hbPG}_{3}$ particles before sintering (top view $a$, side view $b$ ) and after sintering (top view $c$, side view d). Optical micrograph of a silicon wafer coated with $\mathrm{hbPG}_{3}-b-\mathrm{POM}_{120^{-}}$ $b-h_{P P G}$ particles before (e) and after ( $f$ ) sintering. naked eye as an opaque layer before sintering. After the sintering process a transparent and colorless polymer film had been generated that may find application in high resistant coatings for special applications.

SEM images of the obtained polymer films after the short sintering procedure are shown in Fig. 5 in top and side view. The thickness of the films is in the $\mu \mathrm{m}$ range.

The thin films were investigated via static contact angle measurements at the liquid/vapor interface against water to

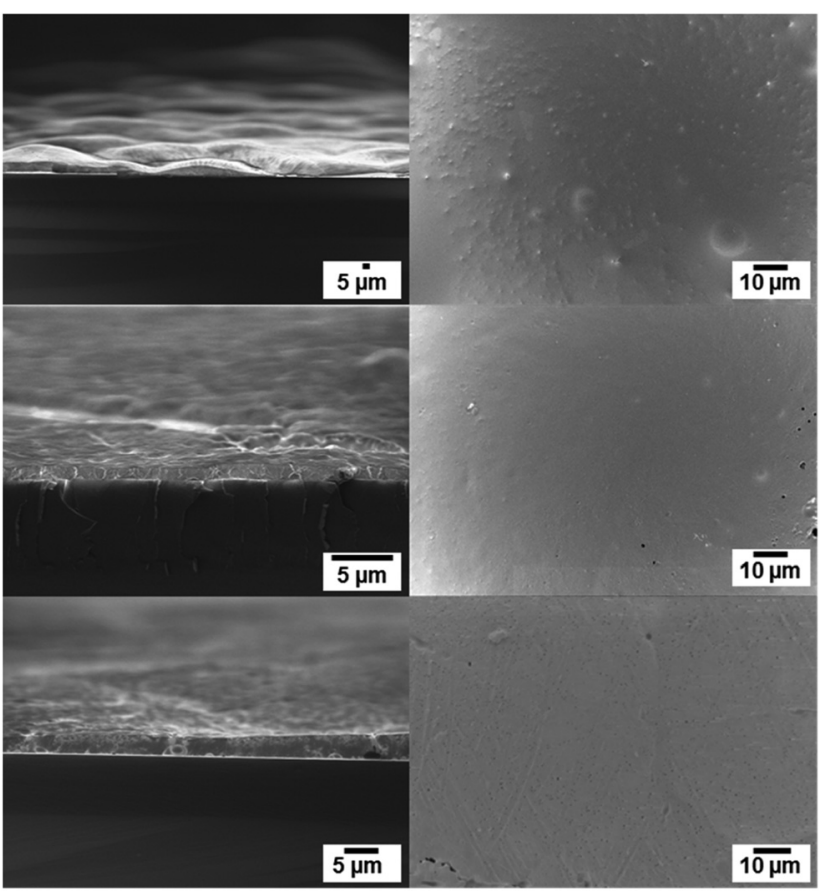

Fig. 5 SEM images of a drop-casted dispersion of $\mathrm{POM} \mathrm{hbPG}_{3}-b$ $\mathrm{POM}_{120}-b-\mathrm{hbPG}_{3}$ and $\mathrm{hbPG}_{7}-b-\mathrm{POM}_{120}-b-\mathrm{hbPG}_{7}$ after sintering. Side view (left side) and top view (right side) of the film.

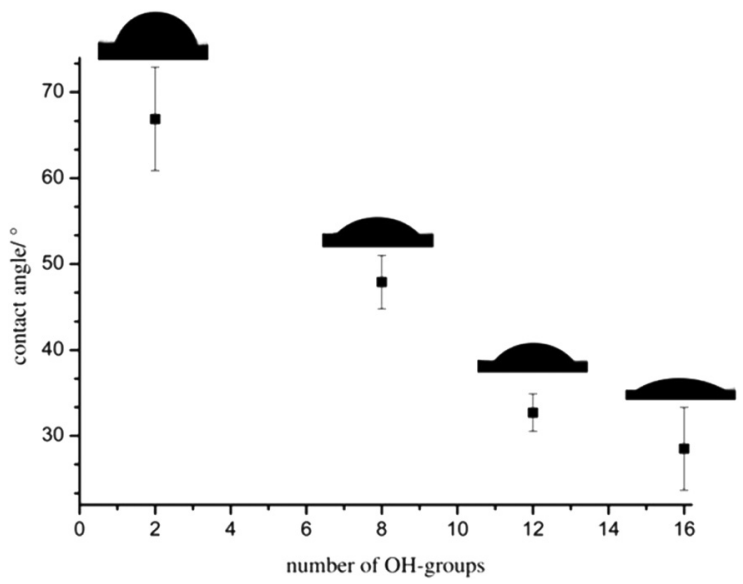

Fig. 6 Static contact angles of sintered polymer films vs. the number of hydroxyl groups of the polymers $\mathrm{POM} \mathrm{hbPG}_{3}-b-\mathrm{POM}_{120}-b-\mathrm{hbPG}_{3}$, hbPG $5-b-\mathrm{POM}_{120}-b-\mathrm{hbPG}$ and $\mathrm{hbPG}_{7}-b-\mathrm{POM}_{120}-b-\mathrm{hbPG}_{7}$. 
analyze the influence of the hbPG-blocks on the film properties. The contact angles decrease from 67 to $29^{\circ}$ for increasing hydroxyl groups from 2 to 16. Fig. 6 summarizes the contact angle $v s$. the number of hydroxyl groups of the polymers. A clear trend to lower contact angles with increasing number of hydroxyl groups is observable enabling the adjustment of the polarity and wettability of the surfaces. This adjustability of the hydrophilicity by varying the hbPG-block size and accompanying the number of hydroxyl groups opens manifold possibilities for the use of POM. In combination with the easy handling of the aqueous nanoparticles dispersions, this approach exhibits promising possibilities for POM as a very important engineering plastic, e.g., in shock-proof coatings.

\section{Conclusions}

This work presents a novel approach for facile processing and film formation of the highly insoluble material POM. An organic or aqueous miniemulsion can be used in order to handle POM in the form of nanoparticles. In addition to POM, also amphiphilic nonlinear copolymers with branched oligoor polyglycerol have been investigated in order to tune the hydrophilicity of the resulting films. Hyperbranched-linearhyperbranched ABA triblock copolymers from hyperbranched polyglycerol (hbPG) and linear poly(oxymethylene) (POM) were synthesized.

The combination of the solvent evaporation process with the miniemulsion technique was used to form nanoparticle dispersions in cyclohexane or water. Dispersions of these nanoparticles were coated on a silicon surface and sintering lead to film formation with film thicknesses in the $\mu \mathrm{m}$ range. Contact angle measurements of these films show a strong dependency of the hydrophilicity of the surface on the number of hydroxyl groups in the polymer backbone.

These nanoparticles could be used for specialty coatings, where the excellent impact and tensile strength, low friction coefficients, low abrasion and high resistance of POM and the hydrophilicity of hbPG on the other hand may be combined. The hydrophilicity of the films can be tuned and further functionalization is an additional option.

\section{Acknowledgements}

M. B. and R. K. thank the Graduate School of Excellence Materials Science in Mainz "MAINZ" (DFG/GSC 266) for financial support. F. R. W. thanks the Max Planck Graduate Center (MPGC) for support.

\section{Notes and references}

1 M. Haubs, K. Kurz and G. Sextro, Polyoxymethylenes, Ullmann's Encyclopedia of Industrial Chemistry, Weinheim, Germany, 2012.
2 K. Weissermel, E. Fischer, K. Gutweiler, H. D. Hermann and H. Cherdron, Angew. Chem., Int. Ed. Engl., 1967, 6, 526-533.

3 G. Kumaraswamy, N. S. Surve, R. Mathew, A. Rana, S. K. Jha, N. N. Bulakh, A. A. Nisal, T. G. Ajithkumar, P. R. Rajamohanan and R. Ratnagiri, Macromolecules, 2012, 45, 5967-5978.

4 S. Lüftl, V.-M. Archodoulaki and S. Seidler, Polym. Degrad. Stab., 2006, 91, 464-471.

5 I. C. Romero-Ibarra, E. Bonilla-Blancas, A. Sánchez-Solís and O. Manero, J. Polym. Eng., 2012, 32, 319-326.

6 M. Sánchez-Soto, S. Illescas, H. Milliman, D. A. Schiraldi and A. Arostegui, Macromol. Mater. Eng., 2010, 295, 846858.

7 N. Vilà Ramirez, M. Sanchez-Soto and S. Illescas, Polym. Compos., 2011, 32, 1584-1592.

8 S. Wacharawichanant, A. Sangkhaphan, N. Sa-Nguanwong, V. Khamnonwat, S. Thongyai and P. Praserthdam, J. Appl. Polym. Sci., 2012, 123, 3217-3224.

9 S. Thomas, A. Paul, A. J. Jose and M. Alagar, Macromol. Symp., 2012, 320, 24-37.

10 K. Pielichowska, J. Appl. Polym. Sci., 2012, 123, 2234-2243.

11 K. Pielichowska, E. Dryzek, Z. Olejniczak, E. Pamuła and J. Pagacz, Polym. Adv. Technol., 2013, 24, 318-330.

12 K. H. Hu, X. G. Hu and X. J. Sun, Appl. Surf. Sci., 2010, 256, 2517-2523.

13 L.-H. Sun, Z.-G. Yang and X.-H. Li, Polym. Eng. Sci., 2008, 48, 1824-1832.

14 K. Hu, J. Wang, S. Schraube, Y. Xu, X. Hu and R. Stengler, Wear, 2009, 266, 1198-1207.

15 L.-H. Sun, Z.-G. Yang and X.-H. Li, Wear, 2008, 264, 693700.

16 G. C. Psarras, S. Siengchin, P. K. Karahaliou, S. N. Georga, C. A. Krontiras and J. Karger-Kocsis, Polym. Int., 2011, 60, 1715-1721.

17 A. Grigalovica, I. Bochkov, R. M. Meri, J. Zicans, J. Grabis, R. Kotsilkova and I. Borovanska, Mater. Sci. Eng., 2012, 38, 12053.

18 S. Wacharawichanant, S. Thongyai, A. Phutthaphan and C. Eiamsam-ang, Polym. Test., 2008, 27, 971-976.

19 T. Kongkhlang, Y. Kousaka, T. Umemura, D. Nakaya, W. Thuamthong, Y. Pattamamongkolchai and S. Chirachanchai, Polymer, 2008, 49, 1676-1684.

20 W. Xu, M. Ge and P. He, J. Appl. Polym. Sci., 2001, 82, 22812289.

21 K. Landfester, Angew. Chem., Int. Ed., 2009, 48, 4488-4507.

22 K. Landfester, Adv. Mater., 2001, 13, 765-768.

23 R. H. Staff, D. Schaeffel, A. Turshatov, D. Donadio, H.-J. Butt, K. Landfester, K. Koynov and D. Crespy, Small, 2013, 9, 3514-3522.

24 K. Landfester, R. Montenegro, U. Scherf, R. Güntner, U. Asawapirom, S. Patil, D. Neher and T. Kietzke, Adv. Mater., 2002, 14, 651-655.

25 A. Musyanovych, J. Schmitz-Wienke, V. Mailänder, P. Walther and K. Landfester, Macromol. Biosci., 2008, 8, 127-139.

26 D. L. Tillier, J. Meuldijk and C. E. Koning, Polymer, 2003, 44, 7883-7890. 
27 A. Thomas, H. Schlaad, B. Smarsly and M. Antonietti, Langmuir, 2003, 19, 4455-4459.

28 R. Klein, C. Schüll, E. Berger-Nicoletti, M. Haubs, K. Kurz and H. Frey, Macromolecules, 2013, 46, 88458852 .

29 F. Wurm and H. Frey, Prog. Polym. Sci., 2011, 36, 1-52.

30 F. Wurm, J. Nieberle and H. Frey, Macromolecules, 2008, 41, 1184-1188.
31 F. Wurm, U. Kemmer-Jonas and H. Frey, Polym. Int., 2009, 58, 989-995.

32 R. Vieweg, Kunststoff Handbuch, Grundlagen, 1. Auflage, München, 1975.

33 A. Sunder, H. Türk, R. Haag and H. Frey, Macromolecules, 2000, 33, 7682-7692.

34 R. H. Staff, I. Lieberwirth, K. Landfester and D. Crespy, Macromol. Chem. Phys., 2012, 213, 351-358. 